\title{
Effect of Water Regime and Antitranspirants Foliar on Production and Yield of Cabbage in Summer Season
}

\author{
A. Y. Ramadan* and M. M. Omar** \\ *Vegetable Research Department, Horticultural Research Institute, Agricultural \\ Research Center, Giza and ${ }^{* *}$ Soil Department, Faculty of Agriculture, Mansoura \\ University, Egypt
}

\begin{abstract}
$\mathbf{F}$ IELD experiments were performed during the summer seasons of 2014 and 2015 at El-Baramon Experimental Farm, Dakahlia Governorate, Egypt to study the effect of irrigation levels and some foliar applications of antitranspirants on the growth, yield, water use efficiency and NPK content of cabbage grown in clayey-textured soil. Results indicated that frequent irrigation with $100 \%$ replenishment of evaporation losses resulted in the highest fresh and head weight, stem diameter, yield and NPK content. On other hand, the flowering and dry matter percentage recorded a negative liner. Water use efficiency recorded high value with either $100 \%$ or $80 \%$ replenishment of evaporation losses. Also, the antitranspirants foliar application increased significantly all characters under study. Whereas, the beneficial effect of antitranspirants foliar application can be arranged as follows: $\mathrm{CaCO}_{3}>$ kaolin $>\mathrm{K}_{2} \mathrm{SO}_{4}>$ plastic film $>$ mineral oil as compared with the untreated plants. Apparently, plant growth, yield and NPK content increased with evaporation replenishment at $80 \%$ level and with the application of antitranspirants and decreased with increasing water stress $(60 \%)$. Generally, it could be concluded that the treatment of $80 \%$ replenishment of evaporation within $3 \% \mathrm{CaCO}_{3}$ was the best combination and it could be recommended for cabbage (c.v. Balady) grown under similar field conditions in order to get optimum yield and to save irrigation water.
\end{abstract}

Keywords: Cabbage, Irrigation, Water stress, Antitranspirants.

\section{Introduction}

Cabbage (Brassica oleracea var. capitata L.) is a very important leafy vegetable crop for fresh and cooked consumption in Egypt. Cabbage leaves have a high alkalinity as a food and contain many nourishing minerals and vitamins. These useful characteristics have made cabbage the most popular leaf-vegetable crop (Haraand Sonoda, 1979). It is cultivated over the year in Egypt, the cultivated area during the year 2015 was 44993 feddan, producing 555396 ton with an average yield of 12.344 ton fed $^{-1}$ (according to the Ministry of Agriculture, Extension Department).

Nowadays, Egypt faces a problem in the amounts of irrigation water because the Egyptian water budget is fixed, thus the main step of the Egyptian strategy is increasing crop productivity from unit area with the lowest irrigation water to save irrigation water. In crop production, instead of achieving maximum yield from a unit area by full irrigation, water productivity can be optimized within the concept of deficit irrigation (Fereres \&
Soriano, 2007 and Geerts \& Raes, 2009). Water stress is one of the most important environmental limitations affecting the plant growth and productivity (Tamer, 2014), whereas, water stress increases the formation of reactive oxygen species causing damage to proteins, membrane lipids and cellular components (Apel and Hirt , 2004). Drought inhibits photochemical events and decreases the activity of enzymes in Calvin cycle (Bruce et al., 2002). Cabbage is classified among vegetables crops with intermediate susceptibility to water stress (Smittle et al., 1994, Kage et al., 2004 and $\mathrm{Xu} \&$ Leskover, 2014). Because of increasing water cost, management of crop water productivity/water use efficiency for crops into semi-arid area is important for a better profitability (Geerts \& Raes 2009 and Xu \& Leskover, 2014).

Irrigation should be managed concurrently to maximize yield, quality and irrigation efficiency for cabbage (McKeown et al., 2010). Increasing the water application, increased significantly cabbage head diameter, head weight, leaf weight and marketable yield (Sammis et al., 1988 \& 1989, 
AbdelRahman et al., 1994, Parmar et al., 1999, AL-Rawahy et al., 2004, McKeown et al., 2010 and Ibrahim et al., 2011). Agricultural scientists suggested the potential use of antitranspirants to enhance the yield of agronomic crops exposed to water stress during growth (Nickell, 1982 and Fenton, et al., 1982). Antitranspirantsis most important tools to reach more and better yield in vegetable by foliar application, which aimed to protect the plants from unsuitable climatic condition (Tambussi and Bort, 2007). El-Afifi et al. (2013) found that antitranspirants reduced the water losses during the vegetative growth period either before or after fruits harvesting. Antitranspirant compounds are applied to foliage to limit the water losses. They include both filmforming and stomata closing compounds, able to increase the leaf resistance to watervapor losses thus improving plant water use to assimilate carbon and in turn, the production of biomass or yield (Tambussi and Bort, 2007).Moreover, filmforming polymers have been previously employed in crop protection against fungal pathogens, thus revealing themselves as promising non-toxic fungicides (Walters, 1992 and Sutherland \& Walters, 2001 , 2002), particularly in post-harvest treatments, where they can also ameliorate the fruit quality under storage conditions (Sivakumar et al., 2005).

Foliar antitranspirants sprays may reduce the rate of transpiration in three ways: 1 . Reflecting materials, reduce absorption of radiant energy and thereby reduce leaf temperatures (Gaballah and Moursy, 2004) and transpiration; 2. Filmforming antitranspirants such as emulsions of wax, latex or plastics dry on foliage to form a thin transparentfilm, providing a physical barrier over some, not all stomata. This hinders escape of water vapor from leaves and also reduces water losses through guttation. 3. Certain chemical compounds such as calcium carbonate can prevent stomata from opening fully by affecting stomatal guard cells, decreasing losses of water vapor (Davenport et al., 1974, Han, 1990 and Steinberg et al., 1990). The purpose of this study is an attempt to improve cabbage productivity under water stress during the summer season using some antitranspirants to reduce water losses under Egyptian conditions.

\section{Materials and Methods}

The field experiment was performed at El-Baramon Experimental Farm, Dakahlia Governorate, Egypt to study the effect of some antitranspirants on maximizing plant tolerance against drought stress. Cabbage (Brassica oleracea var. capitata L. c.v. Balady) was chosen for their susceptibility to water deficient studies. In a split plot design with three replicates, three irrigation regimes $\left(\left(\mathrm{A}_{1}\right)\right.$ conventional irrigation, $\left(A_{2}\right) 80 \%$ and $\left(A_{3}\right) 60 \%$ of the field capacity) were presented in the main plots. While, foliar antitranspirants treatments were allocated in sub plots as follows:

1. $3 \%$ Kaolin (aluminum silicate).

2. $3 \%$ plastic film (100\% acrylic).

3. $3 \%$ Calcium carbonate $\mathrm{CaCO}_{3}$

4. $3 \%$ Potassium sulphate $\mathrm{K}_{2} \mathrm{SO}_{4}$

5. $3 \%$ Mineral oil

6. Control (water spray).

Seedlings were transplanted in the summer season of 2014 and 2015 (24 $4^{\text {th }}$ and $27^{\text {th }}$ May, respectively). In a clayey-textured soil, seedlings were transplanted in plots $(10 \mathrm{~m}$ long with two rows for each). The distance between each row was $80 \mathrm{~cm}$ and the distance between plants were $50 \mathrm{~cm}$. According to the official recommendation for cabbage crop, ammonium sulphate $(20.6 \% \mathrm{~N})$, calcium super phosphate $\left(15.5 \% \quad \mathrm{P}_{2} \mathrm{O}_{5}\right)$ and potassium sulphate $\left(48 \% \mathrm{~K}_{2} \mathrm{O}\right)$ were applied at 100,75 and 50 , respectively. Super phosphate was added in two doses, the $1^{\text {st }}(45 \mathrm{~kg} \mathrm{P} \mathrm{O} /$ fed $)$ was broadcasted during bed preparation ${ }^{2}$ and the $2^{\text {nd }}\left(30 \mathrm{~kg} \mathrm{P} \mathrm{O}_{5} /\right.$ fed $)$ was banded-side dressed after 30 days from planting. Nitrogen and potassium were added side dressed at two equal doses after 30 and 50 days from planting, respectively. Weeds were controlled by hand. The other agricultural practices were carried out according to the recommendations of Ministry of Agriculture.

\section{Irrigation treatments optimization}

Irrigation was applied using conventional furrow irrigation system. At the beginning of the experiment, all treatments were fully irrigated until 50 days from transplanting. In this period, irrigation was adjusted to reach the field capacity, and irrigation was optimized to reach the assumed field capacity (10-12 days) based on meteorological conditions (Ibrahim et al., 2011). Thereafter, all experimental plots were divided into three main groups, the first was irrigated at 10 days interval (7 irrigations) and the second was irrigated at 15 days interval (5 irrigations), while the third was irrigated at 20 days interval (3 irrigations) for I1, I2 and I3, respectively .

Antitranspirants was applied as liquid concentrates and diluted with water. It was applied 
by hand spray gun. Plants were treated twice with antitranspirants applications on both sides of outer leaves in percentage as mentioned previously. The first application was sprayed at 50 days after seedling, and the second one was applied one month later. Potential evepotranspiration (ETo) was calculated (Table 1) according to the FAO PenmanMontieth method Israelson and Hansen (1962) as follows:

$\mathrm{ETO}=\frac{0.408 \Delta(R n-G)+y u 2(e a-e d) \frac{900}{T c+273.15}}{\Delta+y(1+0.34 u 2)}$

Where:

ETO $=$ reference evapotranspiration (grass) (mm/day)

$\mathrm{Rn}=$ net radiation at crop surface $(\mathrm{MJ} / \mathrm{m} 2 /$ day $)$

$\mathrm{G}=$ Soil heat flux $(\mathrm{MJ} / \mathrm{m} 2 /$ day $)$

$\mathrm{Tc}=$ average temperature $\left(\mathrm{C}^{\circ}\right)$

$\mathrm{U} 2=$ wind speed at $2 \mathrm{~m}$ height $(\mathrm{m} / \mathrm{s})$

ea $=$ saturation vapour pressure $(\mathrm{kpa})$

ed $=($ actual vapour pressure $(\mathrm{kPa})$

$\Delta=$ slope of the saturation vapour pressure curve at mean air

$\mathrm{y}=$ psychrometric constant $(\mathrm{kPa} / \mathrm{C} \bullet)$.

A random sample of five plants from each experimental plot was taken at the marketing stage (120 days after seedling) to estimate fresh weight, head weight and dry weight as well as stem diameter. Flowering percentage was estimated by collecting the flowering plant per plot. At the same time, the total yield/plot was estimated by the whole plants.

Random samples of outer and inner leaves from five heads in each plot were chosen, oven dried at $70^{\circ} \mathrm{C}$ and ground for the determination of NPK content.

1. Total nitrogen (\%) was determined according to the methods described by Pregle (1945), using micro-Kjeldahl.

2. Total phosphorus (\%) was determined colorimetrically using the chlorostannus reduce molybdo phosphoric blue color method in sulphoric system as described by Jackson (1967).

3. Potassium (\%) was determined using a flame photometer according to Black (1965).

The monthly mean temperature and relative humidity during the growth period in 2014 and 2015 seasons were presented in Table 2.

\section{Water use efficiency (WUE)}

It is defined as the weight of marketable crop produced per the volume unit of water consumed by plants or the evapotranspiration quantity. The crop water use efficiency was computed for the different treatments by dividing the yield $(\mathrm{kg})$ on units of evapotranspiration expressed as cubic meters of water (Abd El Rasool et al., 1971). It was calculated by the following formula:

$\mathrm{WUE}=$

Yield $(\mathrm{kg} / \mathrm{fed})$

Water consumptive use $\left(\mathrm{m}^{3} / \mathrm{fed}\right)$

Representative samples were collected from the surface layer $(0-45 \mathrm{~cm})$ of the experimental soil and analyzed for some physical and chemical properties as shown in Table 3.

TABLE 1. Computed daily $(\mathrm{mm})$, monthly $(\mathrm{cm})$ and seasonal $\left(\mathrm{m}^{3}\right)$ potential evapotranspiration $($ ETo) in both seasons

\begin{tabular}{ccccccc}
\hline \multirow{2}{*}{ Months } & \multicolumn{3}{c}{ First season 2014 } & \multicolumn{3}{c}{ Second season 2015 } \\
\cline { 2 - 7 } & $\mathbf{m m} / \mathbf{d a y}$ & $\mathbf{c m} / \mathbf{m o n t h}$ & $\mathbf{m}^{\mathbf{3} / \mathbf{m o n t h}}$ & $\mathbf{m m} / \mathbf{d a y}$ & $\mathbf{c m} / \mathbf{m o n t h}$ & $\mathbf{m}^{3} / \mathbf{m o n t h}$ \\
\hline June & 5.91 & 17.73 & 744.66 & 5.94 & 17.82 & 748.44 \\
July & 5.99 & 18.57 & 779.89 & 6.01 & 18.63 & 782.50 \\
August & 5.86 & 18.16 & 762.97 & 5.91 & 18.32 & 769.48 \\
\hline $\mathrm{m}^{3}$ /season & & 2287.5 & & & 2300.4 & \\
\hline
\end{tabular}


TABLE 2. The monthly mean temperature and relative humidity during growth period in 2014 and 2015 seasons

\begin{tabular}{|c|c|c|c|c|c|c|c|c|c|c|c|c|}
\hline \multirow{3}{*}{$\stackrel{\Xi}{\stackrel{\Xi}{\Xi}}$} & \multicolumn{6}{|c|}{ Temperature $\left({ }^{\circ} \mathrm{C}\right)$} & \multicolumn{6}{|c|}{ Relative humidity (\%) } \\
\hline & \multicolumn{3}{|c|}{2014} & \multicolumn{3}{|c|}{2015} & \multicolumn{3}{|c|}{2014} & \multicolumn{3}{|c|}{2015} \\
\hline & Max. & Min. & Mean & Max. & Min. & Mean & Max. & Min. & Mean & Max. & Min. & Mean \\
\hline May & 29.03 & 16.03 & 22.53 & 30.12 & 16.68 & 23.40 & 83.45 & 42.55 & 63.00 & 85.34 & 44.32 & 64.83 \\
\hline Jun. & 30.80 & 19.86 & 25.33 & 31.03 & 19.23 & 25.13 & 82.63 & 42.46 & 62.545 & 85.45 & 43.33 & 64.39 \\
\hline Jul. & 33.17 & 22.08 & 27.63 & 31.56 & 21.80 & 26.68 & 83.46 & 42.13 & 62.795 & 86.11 & 44.56 & 65.35 \\
\hline Aug. & 33.82 & 22.62 & 28.22 & 32.45 & 21.08 & 26.77 & 82.53 & 41.62 & 62.075 & 83.91 & 43.71 & 63.81 \\
\hline
\end{tabular}

TABLE 3. Analytical data of the experimental soil during both seasons of 2014 and 2015

(a) Mechanical and chemical analysis

\begin{tabular}{lcclcc}
\hline \multicolumn{1}{c}{ Soil character } & $\mathbf{2 0 1 4}$ & $\mathbf{2 0 1 5}$ & \multicolumn{1}{c}{ Soil character } & $\mathbf{2 0 1 4}$ & $\mathbf{2 0 1 5}$ \\
\hline Sand & 26.50 & 27.55 & EC. mmhos cm ${ }^{-1}$ at $25^{\circ} \mathrm{C}$ & 0.87 & 0.90 \\
Silt & 35.68 & 35.71 & PH (1:2.5 soil : water susp.) & 8.19 & 8.14 \\
Clay & 37.82 & 36.74 & Available N (ppm) & 42.50 & 43.01 \\
Texture class & Clay & Clay & Available P (ppm) & 5.02 & 5.08 \\
O.M \% & 1.52 & 1.49 & Available K (ppm) & 180 & 198 \\
$\mathrm{CaCO}_{3} \%$ & 2.14 & 2.16 & & & \\
\hline
\end{tabular}

(b) Hydrophysical analysis

\begin{tabular}{ccccccccc}
\hline $\begin{array}{c}\text { Constants } \\
\text { depth } \\
(\mathbf{c m})\end{array}$ & $\begin{array}{c}\text { Field capacity } \\
\mathbf{( \% )}\end{array}$ & \multicolumn{2}{c}{$\begin{array}{c}\text { Wilting point } \\
\mathbf{( \% )}\end{array}$} & \multicolumn{2}{c}{$\begin{array}{c}\text { Available water } \\
\text { (\%) }\end{array}$} & \multicolumn{2}{c}{$\begin{array}{c}\text { Bulk density } \\
\text { (g/cm3) }\end{array}$} \\
\hline $0-15$ & $\mathbf{2 0 1 4}$ & $\mathbf{2 0 1 5}$ & $\mathbf{2 0 1 4}$ & $\mathbf{2 0 1 5}$ & $\mathbf{2 0 1 4}$ & $\mathbf{2 0 1 5}$ & $\mathbf{2 0 1 4}$ & $\mathbf{2 0 1 5}$ \\
\hline $15-30$ & 41.6 & 40.6 & 20.3 & 20.4 & 22.2 & 23.0 & 1.3 & 1.5 \\
$30-45$ & 40.5 & 40.8 & 20.8 & 20.7 & 22.9 & 23.1 & 1.5 & 1.6 \\
& 41.7 & 41.9 & 21.1 & 20.9 & 23.5 & 23.0 & 1.3 & 1.3
\end{tabular}

Data were subjected to analysis of variance using the COSTAT statistical software package. Analysis of variance showed significant F-test for treatment effects, Duncan's multiple range tests was applied to compare the means at $\mathrm{P} \leq 0.05$ (Steel and Torrie, 1998)

\section{Results and Discussion}

Growth and yield

Data in Table 4 and 5 indicate that, the mean values of fresh weight, head weight and yield decreased significantly with decreasing evaporation replenishment during both seasons. While, dry matter and flowering percentage increased with the same treatments. With regard to the effect of foliar spraying of antitranspirants, all growth parameters were significantly increased in response to spraying all foliar application as follows: $\mathrm{CaCO}_{3}>$ kaolin $>\mathrm{K}_{2} \mathrm{SO}_{4}>$ plastic film $>$ mineral oil as compared with the untreated plants. The highest significant values of the parameters above were recorded with spraying plants by $\mathrm{CaCO}_{3} \quad(3 \%)$ with evaporation replenishment $(100 \%$ or $80 \%)$. Whereas, the differences reach to the level of significance in the case of using $\mathrm{CaCO}_{3}$ with $80 \%$ evaporation replenishment compared with untreated plants under evaporation replenishment $100 \%$. These increases were true in the two seasons of the experiment. It could be suggested that increasing water quantity applied to plant led to keep higher moisture content in the soil and this in turn might favor the plant metabolism that leads to increase the plant growth characters and to produce higher yield. These results were clearly coincided with that obtained by Byari and A1-Sayed (1999) on tomato, Abbas et al. (1999) on rape seed, Abbas (2007) on eggplant, Abdel-Aal et al. (2008) on eggplant and Bahawireth (2011). 
TABLE 4. Effect of water regime and antitranspirants foliar applications on stem length, fresh and dry weight of cabbage during 2014 and 2015 seasons

\begin{tabular}{|c|c|c|c|c|c|c|c|}
\hline \multirow{2}{*}{\multicolumn{2}{|c|}{ Treatments }} & \multicolumn{2}{|c|}{ Fresh weight (kg) } & \multicolumn{2}{|c|}{ Head weight (kg) } & \multicolumn{2}{|c|}{ Dry matter $(\%)$} \\
\hline & & 2014 & 2015 & 2014 & 2015 & 2014 & 2015 \\
\hline & & & & \multicolumn{4}{|c|}{ Mean values as affected by water regime } \\
\hline \multirow{3}{*}{\multicolumn{2}{|c|}{$\begin{array}{l}\text { Irrigation (I1) E.R } 100 \% \\
\text { Irrigation (I2) E.R } 80 \% \\
\text { Irrigation (I3) E.R } 60 \%\end{array}$}} & $3.73 \mathrm{a}$ & $3.26 \mathrm{a}$ & $3.52 \mathrm{a}$ & $3.07 \mathrm{a}$ & $6.52 \mathrm{c}$ & $8.04 \mathrm{a}$ \\
\hline & & $3.19 \mathrm{~b}$ & $2.60 \mathrm{~b}$ & $2.97 \mathrm{~b}$ & $2.45 \mathrm{~b}$ & $7.53 \mathrm{~b}$ & $7.42 \mathrm{~b}$ \\
\hline & & $2.17 \mathrm{c}$ & $2.04 \mathrm{c}$ & $2.04 \mathrm{c}$ & $1.89 \mathrm{c}$ & $8.03 \mathrm{a}$ & $6.42 c$ \\
\hline \multicolumn{8}{|c|}{ Mean values as affected by antitranspirants foliar applications } \\
\hline & Kaolin & $3.43 \mathrm{~b}$ & $2.86 \mathrm{~b}$ & $3.26 \mathrm{~b}$ & $2.68 \mathrm{~b}$ & $7.76 \mathrm{~b}$ & $7.57 \mathrm{bc}$ \\
\hline & Plastic film & $2.99 \mathrm{c}$ & $2.56 \mathrm{~d}$ & $2.71 \mathrm{~d}$ & $2.40 \mathrm{~d}$ & $7.08 \mathrm{c}$ & $7.30 \mathrm{c}$ \\
\hline & $\mathrm{CaCO}_{3}$ & $3.66 \mathrm{a}$ & $3.16 \mathrm{a}$ & $3.43 \mathrm{a}$ & $2.95 \mathrm{a}$ & $8.42 \mathrm{a}$ & $8.63 \mathrm{a}$ \\
\hline & $\mathrm{K}_{2} \mathrm{SO}_{4}$ & $3.09 \mathrm{c}$ & $2.70 \mathrm{c}$ & $2.02 \mathrm{c}$ & $2.53 \mathrm{c}$ & $7.54 \mathrm{~b}$ & $7.70 \mathrm{~b}$ \\
\hline & Mineral Oil & $2.82 \mathrm{~d}$ & $2.44 \mathrm{e}$ & $2.68 \mathrm{~d}$ & $2.31 \mathrm{~d}$ & $6.90 \mathrm{c}$ & $6.45 \mathrm{~d}$ \\
\hline & Without & $2.20 \mathrm{e}$ & $2.07 \mathrm{f}$ & $2.07 \mathrm{e}$ & $1.96 \mathrm{e}$ & $6.46 \mathrm{~d}$ & $6.10 \mathrm{e}$ \\
\hline \multirow[t]{2}{*}{$\begin{array}{c}\text { Water } \\
\text { regime }\end{array}$} & \multicolumn{3}{|c|}{ Antitranspirants } & & & & \\
\hline & Kaolin & $4.196 \mathrm{~b}$ & $3.441 \mathrm{~b}$ & $3.941 \mathrm{~b}$ & $3.226 \mathrm{~b}$ & 6.91ghi & $6.62 \mathrm{fg}$ \\
\hline \multirow{5}{*}{$\begin{array}{l}\text { Irrigation } \\
\quad \text { (I1) } \\
\text { E.R } 100 \%\end{array}$} & Plastic film & $3.523 \mathrm{de}$ & $3.123 \mathrm{c}$ & $3.313 \mathrm{de}$ & $2.933 \mathrm{c}$ & $6.46 \mathrm{ij}$ & $6.36 \mathrm{fgh}$ \\
\hline & $\mathrm{CaCO}_{3}$ & $4.481 \mathrm{a}$ & $3.826 \mathrm{a}$ & $4.260 \mathrm{a}$ & $3.563 \mathrm{a}$ & $7.43 \mathrm{def}$ & $7.81 \mathrm{bc}$ \\
\hline & $\mathrm{K}_{2} \mathrm{SO}_{4}$ & $3.863 \mathrm{c}$ & $3.322 \mathrm{~b}$ & $3.673 \mathrm{c}$ & $3.126 \mathrm{~b}$ & $6.77 \mathrm{hi}$ & $6.53 \mathrm{fg}$ \\
\hline & Mineral Oil & $3.322 \mathrm{efg}$ & $3.051 \mathrm{c}$ & $3.122 \mathrm{ef}$ & $2.873 \mathrm{~cd}$ & $6.23 \mathrm{j}$ & $5.87 \mathrm{~h}$ \\
\hline & Without & $3.011 \mathrm{hi}$ & $2.833 \mathrm{~d}$ & $2.843 \mathrm{gh}$ & $2.721 \mathrm{de}$ & $5.33 \mathrm{k}$ & $5.33 \mathrm{i}$ \\
\hline \multirow{6}{*}{$\begin{array}{l}\text { Irrigation } \\
\quad \text { (I2) } \\
\text { E.R } 80 \%\end{array}$} & Kaolin & $3.473 \mathrm{df}$ & $2.820 \mathrm{~d}$ & $3.316 \mathrm{de}$ & $2.650 \mathrm{ef}$ & $8.11 b c$ & $7.87 \mathrm{bc}$ \\
\hline & Plasticfilm & $3.211 \mathrm{fgh}$ & $2.506 \mathrm{fg}$ & 2.706 hi & $2.336 \mathrm{~g}$ & $7.45 \mathrm{de}$ & $7.62 \mathrm{~cd}$ \\
\hline & $\mathrm{CaCO}_{3}$ & $3.693 \mathrm{~cd}$ & $3.096 \mathrm{c}$ & $3.456 \mathrm{~cd}$ & $2.926 \mathrm{c}$ & $8.78 \mathrm{a}$ & $8.86 \mathrm{a}$ \\
\hline & $\mathrm{K}_{2} \mathrm{SO}_{4}^{3}$ & $3.286 \mathrm{efg}$ & $2.726 \mathrm{de}$ & $3.121 \mathrm{ef}$ & $2.553 \mathrm{f}$ & $7.69 \mathrm{cde}$ & $7.75 b c$ \\
\hline & Mineral Oil & $3.143 \mathrm{fgh}$ & $2.330 \mathrm{~g}$ & $3.013 \mathrm{fg}$ & $2.233 \mathrm{gh}$ & $6.98 \mathrm{fgh}$ & $6.24 \mathrm{gh}$ \\
\hline & Without & $2.363 \mathrm{k}$ & $2.133 \mathrm{~h}$ & $2.240 \mathrm{i}$ & $2.003 \mathrm{i}$ & $6.21 \mathrm{j}$ & $6.21 \mathrm{gh}$ \\
\hline \multirow{6}{*}{$\begin{array}{l}\text { Irrigation } \\
\quad \text { (I3) } \\
\text { E.R } 60 \%\end{array}$} & Kaolin & $2.636 \mathrm{j}$ & $2.340 \mathrm{~g}$ & $2.531 \mathrm{i}$ & $2.173 \mathrm{~h}$ & $8.27 \mathrm{~b}$ & $8.23 \mathrm{~b}$ \\
\hline & Plasticfilm & $2.253 \mathrm{kl}$ & $2.066 \mathrm{~h}$ & $2.116 \mathrm{jk}$ & $1.930 \mathrm{ij}$ & $7.34 \mathrm{efg}$ & $7.94 b c$ \\
\hline & $\mathrm{CaCO}_{3}$ & $2.803 \mathrm{ij}$ & $2.560 \mathrm{ef}$ & $2.596 \mathrm{i}$ & $2.361 \mathrm{~g}$ & $9.06 \mathrm{a}$ & $9.23 \mathrm{a}$ \\
\hline & $\mathrm{K}_{2} \mathrm{SO}_{4}$ & $2.116 \mathrm{kl}$ & $2.053 \mathrm{~h}$ & $1.973 \mathrm{k}$ & $1.916 \mathrm{ij}$ & $8.15 \mathrm{~b}$ & $8.83 \mathrm{a}$ \\
\hline & Mineral Oil & 2.0211 & $1.960 \mathrm{~h}$ & $1.910 \mathrm{k}$ & $1.826 \mathrm{j}$ & $7.49 \mathrm{de}$ & $7.25 \mathrm{de}$ \\
\hline & Without & $1.236 \mathrm{~m}$ & $1.246 \mathrm{i}$ & 1.1331 & $1.163 \mathrm{k}$ & $7.84 \mathrm{bcd}$ & $6.77 \mathrm{ef}$ \\
\hline
\end{tabular}

Values in each column followed by the same letters are not significantly different at $P \leq 0.05$.

*E.R: Evaporation replenishment

Water use efficiency

The full irrigation treatment (I1) recorded the highest WUE value giving its highest produced yield. Meanwhile, I3 treatment recorded the lowest WUE value (Table 5). Concerning the effect of antitranspirants treatments, data in Table 5 illustrated that the foliar spraying with $\mathrm{CaCO}_{3}$ recorded the highest WUE values followed by kaolin treatment based on their beneficial effect on reducing the hazardous effect of water stress. On the other hand, the control treatment (without foliar spraying of antitransoirants) recorded the lowest WUE. Results indicated that the foliar application of antitranspirant under water stress led to a significant improvement in WUE. In general, leafy vegetables such as cabbage need suitable quantity of water to produce normal yield. However, under water stress conditions, the produced yield showed progressive reduction following the considerable losses of water contents. In this respect, Frank and Viets (1967) stated that growing plants in fertile soil can meet its needs for more nutrients when water conditions are more favorable. Therefore, the decrease of nutrients content in cabbage plant at $60 \%$ WHC may be due to redacting the solubility of mineral in the soil; hence movement of cations to root is reduced. 
TABLE 5. Effect of water regime and antitranspirants foliar applications on fresh and dry weight as well as water use efficiency of cabbage during 2014 and 2015 seasons

\begin{tabular}{|c|c|c|c|c|c|c|c|}
\hline \multirow{2}{*}{\multicolumn{2}{|c|}{ Treatments }} & \multicolumn{2}{|c|}{ Flowering (\%) } & \multicolumn{2}{|c|}{ Yield/plot (ton) } & \multicolumn{2}{|c|}{$* *$ WUE $\left(\mathrm{kg} \cdot \mathrm{m}^{-3}\right)$} \\
\hline & & 2014 & 2015 & 2014 & 2015 & 2014 & 2015 \\
\hline & & & & & \multicolumn{3}{|c|}{ Mean values as affected by water regime } \\
\hline \multicolumn{2}{|c|}{ Irrigation (I1) E.R 100\% } & $8.61 \mathrm{c}$ & $6.53 \mathrm{c}$ & $0.194 \mathrm{a}$ & $0.182 \mathrm{a}$ & $12.74 \mathrm{a}$ & $11.90 \mathrm{a}$ \\
\hline \multicolumn{2}{|c|}{ Irrigation (I2) E.R 80\% } & $11.93 \mathrm{~b}$ & $10.28 \mathrm{~b}$ & $0.172 \mathrm{~b}$ & $0.158 \mathrm{~b}$ & $11.28 \mathrm{~b}$ & $10.30 \mathrm{~b}$ \\
\hline \multicolumn{2}{|c|}{ Irrigation (I3) E.R 60\% } & $16.05 \mathrm{a}$ & $15.27 \mathrm{a}$ & $0.131 \mathrm{c}$ & $0.121 \mathrm{c}$ & $8.63 \mathrm{c}$ & $7.94 \mathrm{c}$ \\
\hline \multicolumn{8}{|c|}{ Mean values as affected by antitranspirants foliar applications } \\
\hline \multicolumn{2}{|c|}{ Kaolin } & $8.78 \mathrm{~d}$ & $8.08 \mathrm{~d}$ & $0.182 \mathrm{~b}$ & $0.179 \mathrm{~b}$ & $11.97 \mathrm{~b}$ & $11.70 \mathrm{~b}$ \\
\hline \multicolumn{2}{|r|}{ Plastic film } & $11.08 \mathrm{c}$ & $10.54 \mathrm{c}$ & $0.162 \mathrm{~d}$ & $0.146 \mathrm{~d}$ & $10.63 \mathrm{~d}$ & $9.54 \mathrm{~d}$ \\
\hline \multicolumn{2}{|r|}{$\mathrm{CaCO}_{3}$} & $7.69 \mathrm{e}$ & $6.56 \mathrm{e}$ & $0.201 \mathrm{a}$ & $0.197 \mathrm{a}$ & $13.23 \mathrm{a}$ & $12.88 \mathrm{a}$ \\
\hline \multicolumn{2}{|r|}{$\mathrm{K}_{2} \mathrm{SO}_{4}$} & $11.16 \mathrm{c}$ & $10.38 \mathrm{c}$ & $0.173 \mathrm{c}$ & $0.160 \mathrm{c}$ & $11.40 \mathrm{c}$ & $10.44 \mathrm{c}$ \\
\hline \multicolumn{2}{|r|}{ Mineral Oil } & $16.11 \mathrm{~b}$ & $13.65 \mathrm{~b}$ & $0.153 \mathrm{e}$ & $0.126 \mathrm{e}$ & $10.06 \mathrm{e}$ & $8.25 \mathrm{e}$ \\
\hline \multicolumn{2}{|r|}{ Without } & $18.35 \mathrm{a}$ & $14.94 \mathrm{a}$ & $0.122 \mathrm{f}$ & $0.114 \mathrm{f}$ & $8.03 \mathrm{f}$ & $7.45 \mathrm{f}$ \\
\hline \multicolumn{2}{|l|}{$\begin{array}{l}\text { Water } \\
\text { regime }\end{array}$} & itranspirar & & & & & \\
\hline \multirow{6}{*}{$\begin{array}{l}\text { Irrigation } \\
\quad \text { (I1) } \\
\text { E.R } 100 \%\end{array}$} & Kaolin & $5.92 \mathrm{o}$ & $4.53 \mathrm{~m}$ & $0.219 \mathrm{~b}$ & $0.208 \mathrm{~b}$ & $14.40 \mathrm{~b}$ & $13.58 \mathrm{~b}$ \\
\hline & Plasticfilm & $8.75 \mathrm{~m}$ & $6.83 \mathrm{k}$ & $0.186 \mathrm{~d}$ & $0.177 \mathrm{~d}$ & $12.24 \mathrm{~d}$ & $11.54 \mathrm{~d}$ \\
\hline & $\mathrm{CaCO}_{3}$ & $4.30 \mathrm{q}$ & $3.33 \mathrm{n}$ & $0.242 \mathrm{a}$ & $0.233 \mathrm{a}$ & $15.86 \mathrm{a}$ & $15.21 \mathrm{a}$ \\
\hline & $\mathrm{K}_{2} \mathrm{SO}_{4}^{3}$ & $5.62 \mathrm{p}$ & 6.171 & $0.202 \mathrm{c}$ & $0.190 \mathrm{c}$ & $13.24 \mathrm{c}$ & $12.41 \mathrm{c}$ \\
\hline & Mineral Oil & $12.73 \mathrm{~h}$ & $8.95 \mathrm{i}$ & $0.175 \mathrm{e}$ & $0.152 \mathrm{f}$ & $11.49 \mathrm{e}$ & $9.95 \mathrm{f}$ \\
\hline & Without & $14.35 \mathrm{f}$ & $9.37 \mathrm{hi}$ & $0.140 \mathrm{hi}$ & $0.133 \mathrm{~g}$ & $9.22 \mathrm{hi}$ & $8.69 \mathrm{~g}$ \\
\hline \multirow{6}{*}{$\begin{array}{l}\text { Irrigation } \\
\quad \text { (I2) } \\
\text { E.R } 80 \%\end{array}$} & Kaolin & $8.93 \mathrm{~m}$ & $7.46 \mathrm{j}$ & $0.181 \mathrm{de}$ & $0.195 \mathrm{c}$ & $11.86 \mathrm{de}$ & $12.71 \mathrm{c}$ \\
\hline & Plastic film & 10.791 & $9.52 \mathrm{~h}$ & $0.173 \mathrm{e}$ & $0.147 \mathrm{f}$ & $11.34 \mathrm{e}$ & $9.60 \mathrm{f}$ \\
\hline & $\mathrm{CaCO}_{3}$ & $8.43 n$ & $7.01 \mathrm{jk}$ & $0.206 \mathrm{c}$ & $0.206 \mathrm{~b}$ & $13.55 \mathrm{c}$ & $13.47 \mathrm{~b}$ \\
\hline & $\mathrm{K}_{2} \mathrm{SO}_{4}$ & $11.20 \mathrm{j}$ & $10.09 \mathrm{~g}$ & $0.183 \mathrm{de}$ & $0.164 \mathrm{e}$ & $12.04 \mathrm{de}$ & $10.73 \mathrm{e}$ \\
\hline & Mineral Oil & $14.99 \mathrm{e}$ & $13.38 \mathrm{e}$ & $0.158 \mathrm{f}$ & $0.117 \mathrm{hi}$ & $10.38 \mathrm{f}$ & $7.65 \mathrm{hi}$ \\
\hline & Without & $17.22 \mathrm{c}$ & $14.24 \mathrm{~d}$ & $0.130 \mathrm{ij}$ & $0.117 \mathrm{hi}$ & $8.54 \mathrm{ij}$ & $7.62 \mathrm{hi}$ \\
\hline \multirow{3}{*}{$\begin{array}{c}\text { Irrigation } \\
\text { (I3) }\end{array}$} & Kaolin & $11.49 \mathrm{i}$ & $12.25 \mathrm{f}$ & $0.147 \mathrm{gh}$ & $0.135 \mathrm{~g}$ & $9.66 \mathrm{gh}$ & $8.80 \mathrm{~g}$ \\
\hline & Plastic film & $13.69 \mathrm{~g}$ & $15.28 \mathrm{c}$ & $0.127 \mathrm{j}$ & $0.115 \mathrm{i}$ & $8.32 \mathrm{j}$ & $7.49 \mathrm{i}$ \\
\hline & $\mathrm{CaCO}_{3}$ & 10.341 & $9.35 \mathrm{hi}$ & $0.156 f g$ & $0.153 \mathrm{f}$ & $10.27 \mathrm{fg}$ & $9.97 \mathrm{f}$ \\
\hline \multirow[t]{3}{*}{ E.R $60 \%$} & $\mathrm{~K}_{2} \mathrm{SO}_{4}$ & $16.67 \mathrm{~d}$ & $14.88 \mathrm{c}$ & $0.136 \mathrm{ij}$ & $0.125 \mathrm{gh}$ & $8.91 \mathrm{ij}$ & $8.19 \mathrm{gh}$ \\
\hline & Mineral Oil & $20.61 \mathrm{~b}$ & $18.63 \mathrm{~b}$ & $0.126 \mathrm{j}$ & $0.109 \mathrm{i}$ & $8.30 \mathrm{j}$ & $7.15 \mathrm{i}$ \\
\hline & Without & $23.49 \mathrm{a}$ & $21.22 \mathrm{a}$ & $0.096 \mathrm{k}$ & $0.092 \mathrm{j}$ & $6.33 \mathrm{k}$ & $6.04 \mathrm{j}$ \\
\hline
\end{tabular}

Values in each column followed by the same letters are not significantly different at $P \leq 0.05$.

*E.R: Evaporation replenishment $\quad * *$ WUE: water use efficiency.

Also, antitranspirant products based on stomata close, such as $\mathrm{CaCO}_{3} \mathrm{~K}_{2} \mathrm{SO}_{4}$, are active in heavy water limited environments, where they may improve crop yield which acts on stomatal regulation in an ABA-dependent way, can be more effective in temperate regions, when occasional or episodic drought events occur compared with the film-forming material (Iriti et al., 2009).

\section{NPK concentration}

Nitrogen, phosphorus and potassium concentrations in leaves decreased with replenishing evaporation losses in both seasons (Table 6). Moreover the statistical analysis revealed that the values of the above characters significantly decreased with the irrigation regime treatments. These were true in both seasons. This finding could be attributed to the fact that when soil moisture decreased, the mobility of nutrient in the soil is towered and the rate of nutrients flow to root absorption zone decreased. Similar results were obtained by Mahmoud and Hafiz (2002) on eggplant and Erdal et al. (2007) on tomato. 
TABLE 6. Effect of water regime and antitranspirants foliar applications on NPK percent of cabbage leaves during 2014 and 2015 seasons.

\begin{tabular}{|c|c|c|c|c|c|c|c|}
\hline \multirow{2}{*}{\multicolumn{2}{|c|}{ Treatments }} & \multicolumn{2}{|c|}{ Nitrogen $(\%)$} & \multicolumn{2}{|c|}{ Phosphorus (\%) } & \multicolumn{2}{|c|}{ Potassium (\%) } \\
\hline & & 2014 & 2015 & 2014 & 2015 & 2014 & 2015 \\
\hline \multicolumn{8}{|c|}{ Mean values as affected by water regime } \\
\hline \multicolumn{2}{|c|}{ Irrigation (I1) E.R 100\% } & $3.44 \mathrm{a}$ & $3.29 \mathrm{a}$ & $0.197 \mathrm{a}$ & $0.159 \mathrm{a}$ & $3.31 \mathrm{a}$ & $3.47 \mathrm{a}$ \\
\hline \multicolumn{2}{|c|}{ Irrigation (I2) E.R 80\% } & $3.29 \mathrm{~b}$ & $3.02 \mathrm{~b}$ & $0.176 \mathrm{~b}$ & $0.139 \mathrm{~b}$ & $3.03 \mathrm{~b}$ & $3.21 \mathrm{~b}$ \\
\hline \multicolumn{2}{|c|}{ Irrigation (I3) E.R 60\% } & $2.40 \mathrm{c}$ & $2.44 \mathrm{c}$ & $0.149 \mathrm{c}$ & $0.128 \mathrm{c}$ & $2.32 \mathrm{c}$ & $3.14 \mathrm{c}$ \\
\hline \multicolumn{8}{|c|}{ Mean values as affected by antitranspirants foliar applications } \\
\hline \multicolumn{2}{|c|}{ Kaolin } & $3.08 \mathrm{c}$ & $3.11 \mathrm{~b}$ & $0.194 \mathrm{~b}$ & $0.150 \mathrm{~b}$ & $3.01 \mathrm{~b}$ & $3.32 \mathrm{c}$ \\
\hline \multicolumn{2}{|r|}{ Plasticfilm } & $2.99 \mathrm{~d}$ & $2.82 \mathrm{~d}$ & $0.165 \mathrm{~d}$ & $0.138 \mathrm{c}$ & $2.71 \mathrm{~d}$ & $3.24 \mathrm{~d}$ \\
\hline \multicolumn{2}{|r|}{$\mathrm{CaCO}_{3}$} & $3.46 \mathrm{a}$ & $3.25 \mathrm{a}$ & $0.200 \mathrm{a}$ & $0.157 \mathrm{a}$ & $3.20 \mathrm{a}$ & $3.43 \mathrm{a}$ \\
\hline \multicolumn{2}{|r|}{$\begin{array}{l}\mathrm{K}_{2} \mathrm{SU}_{4} \\
\text { Mineral }\end{array}$} & $3.30 \mathrm{~b}$ & $3.03 \mathrm{c}$ & $0.177 \mathrm{c}$ & $0.148 \mathrm{~b}$ & $2.96 \mathrm{~b}$ & $3.37 \mathrm{~b}$ \\
\hline \multicolumn{2}{|r|}{ Oil } & $2.79 \mathrm{e}$ & $2.69 \mathrm{e}$ & $0.156 \mathrm{e}$ & $0.134 \mathrm{c}$ & $2.64 \mathrm{e}$ & $3.19 \mathrm{~d}$ \\
\hline \multicolumn{2}{|r|}{ Without } & $2.64 \mathrm{f}$ & $2.60 \mathrm{f}$ & $0.152 \mathrm{e}$ & $0.126 \mathrm{~d}$ & $2.80 \mathrm{c}$ & $3.09 \mathrm{e}$ \\
\hline \multirow[t]{2}{*}{$\begin{array}{c}\text { Water } \\
\text { regime }\end{array}$} & Antitranspir & & & & & & \\
\hline & Kaolin & $3.55 \mathrm{c}$ & $3.55 \mathrm{a}$ & $0.211 \mathrm{a}$ & $0.171 \mathrm{a}$ & $3.46 \mathrm{~b}$ & $3.52 \mathrm{bc}$ \\
\hline \multirow{2}{*}{$\begin{array}{l}\text { Irrigation } \\
\text { (I1) }\end{array}$} & Plasticfilm & $3.39 \mathrm{de}$ & $3.14 \mathrm{~d}$ & $0.192 \mathrm{~cd}$ & $0.152 b c$ & $3.18 \mathrm{de}$ & $3.38 \mathrm{~d}$ \\
\hline & $\mathrm{CaCO}_{3}$ & $4.12 \mathrm{a}$ & $3.63 \mathrm{a}$ & $0.219 \mathrm{a}$ & $0.174 \mathrm{a}$ & $3.72 \mathrm{a}$ & $3.65 \mathrm{a}$ \\
\hline \multirow[t]{4}{*}{ E.R $100 \%$} & $\begin{array}{l}\mathrm{K}_{2} \mathrm{SO}_{4} \\
\text { Mineral }\end{array}$ & $3.85 \mathrm{~b}$ & $3.37 \mathrm{~b}$ & $0.197 b c$ & 0.166 a & $3.41 b c$ & $3.56 \mathrm{ab}$ \\
\hline & Oil & $2.87 \mathrm{~g}$ & $3.09 \mathrm{de}$ & $0.186 \mathrm{de}$ & $0.149 \mathrm{bcd}$ & $3.09 \mathrm{f}$ & $3.38 \mathrm{~d}$ \\
\hline & Without & $2.91 \mathrm{~g}$ & $3.01 \mathrm{e}$ & $0.181 \mathrm{ef}$ & $0.145 \mathrm{cde}$ & $3.02 \mathrm{f}$ & $3.35 \mathrm{de}$ \\
\hline & Kaolin & $3.35 \mathrm{e}$ & $3.15 \mathrm{~cd}$ & $0.194 \mathrm{bcd}$ & $0.142 \mathrm{def}$ & 3.10 ef & $3.27 \mathrm{ef}$ \\
\hline \multirow{2}{*}{$\begin{array}{l}\text { Irrigation } \\
\text { (I2) }\end{array}$} & Plasticfilm & $3.19 \mathrm{f}$ & $2.92 \mathrm{f}$ & $0.171 \mathrm{~g}$ & $0.139 \mathrm{ef}$ & $3.08 \mathrm{f}$ & $3.24 \mathrm{fg}$ \\
\hline & $\mathrm{CaCO}_{3}$ & $3.74 \mathrm{~b}$ & $3.23 \mathrm{c}$ & $0.201 \mathrm{~b}$ & $0.155 \mathrm{~b}$ & $3.37 \mathrm{c}$ & $3.44 \mathrm{~cd}$ \\
\hline \multirow[t]{4}{*}{ E.R $80 \%$} & $\begin{array}{l}\mathrm{K}_{2} \mathrm{SO}_{4} \\
\text { Mineral }\end{array}$ & $3.51 \mathrm{~cd}$ & $3.11 \mathrm{~d}$ & $0.176 f g$ & 0.140 ef & $3.19 \mathrm{~d}$ & $3.39 \mathrm{~d}$ \\
\hline & Oil & $3.11 \mathrm{f}$ & $2.91 \mathrm{f}$ & $0.157 \mathrm{~h}$ & $0.134 \mathrm{fg}$ & $2.75 \mathrm{~g}$ & $3.08 \mathrm{ij}$ \\
\hline & Without & $2.87 \mathrm{~g}$ & $2.80 \mathrm{~g}$ & $0.157 \mathrm{~h}$ & 0.127 & $2.70 \mathrm{~g}$ & $2.85 \mathrm{k}$ \\
\hline & Kaolin & $2.35 \mathrm{j}$ & $2.64 \mathrm{~h}$ & $0.177 \mathrm{fg}$ & $0.139 \mathrm{ef}$ & $2.50 \mathrm{~h}$ & 3.17ghi \\
\hline \multirow{2}{*}{$\begin{array}{l}\text { Irrigation } \\
\text { (I3) }\end{array}$} & Plasticfilm & 2.41hig & $2.41 \mathrm{i}$ & $0.134 \mathrm{i}$ & $0.123 \mathrm{~h}$ & $2.20 \mathrm{j}$ & 3.12ghi \\
\hline & $\mathrm{CaCO}_{3}$ & $2.54 \mathrm{hi}$ & $2.89 \mathrm{f}$ & $0.182 \mathrm{ef}$ & $0.143 \mathrm{de}$ & $2.69 \mathrm{~g}$ & $3.22 \mathrm{fg}$ \\
\hline \multirow[t]{3}{*}{ E.R $60 \%$} & $\begin{array}{l}\mathrm{K}_{2} \mathrm{SO}_{4} \\
\text { Mineral }\end{array}$ & $2.55 \mathrm{~h}$ & $2.61 \mathrm{~h}$ & $0.159 \mathrm{~h}$ & $0.138 \mathrm{ef}$ & $2.39 \mathrm{i}$ & $3.18 \mathrm{fgh}$ \\
\hline & Oil & $2.40 \mathrm{ij}$ & $2.08 \mathrm{j}$ & $0.127 \mathrm{ij}$ & $0.121 \mathrm{~h}$ & $2.13 \mathrm{j}$ & $3.12 \mathrm{ghi}$ \\
\hline & Without & $2.16 \mathrm{k}$ & $2.01 \mathrm{j}$ & $0.119 \mathrm{j}$ & $0.106 \mathrm{i}$ & $2.02 \mathrm{k}$ & $3.07 \mathrm{j}$ \\
\hline
\end{tabular}

Values in each column followed by the same letters are not significantly different at $P \leq 0.05$.

*E.R: Evaporation replenishment 
Regarding to the effect of antitranspirants application, data in Table 6 indicated significant increments on NPK concentrations comparing with the untreated plants in both seasons. In this regards foliar spraying of $\mathrm{CaCO}_{3}$ was the superior treatment followed by kaolin and lastly mineral oil. On the other hand, untreated plants recorded the lowest nutrient concentrations in both seasons. The obtained results are consistent with the most previous investigations, which pointed out the same direct correlation between antitranspirants materials and some elemental nutrition in tissues of plant (Moftah , 1997) on soya bean (Yadav and Dashora, 2003).

The effect of the interaction between evaporation replenishment and foliar application of antitranspirants on NPK content was illustrated in Table 6. Data showed that there was a significant increase in N, P and $\mathrm{K}$ content of cabbage leaves in both seasons as affected by foliar applications with different source of antitranspirants under different irrigation intervals in the both seasons. Plants sprayed with $\mathrm{CaCO}_{3}$ at $(3 \%)$ concentration under evaporation replenishment $100 \%$ recorded the highest values of parameters under study compared with other treatments. These results agree with that reported by Abd-El-Aal et al. (2008) on eggplant.

\section{Conclusions}

The results emphasize the importance of application of antitranspirants foliar application under water stress conditions. It is found that, the optimum combinations ( $80 \%$ of field capacity with $3 \% \mathrm{CaCO}_{3}$ concentration as foliar application) for maximum yield accompanied by high water use efficiency. So it could be recommended for cabbage cv. Balady grown under similar field conditions in order to get a higher economical yield and to save irrigation water.

\section{References}

Abbas, F. A., EL-Eman, M. A. and Anton, N. A. (1999) Effect of irrigation intervals on two rape seed varieties. Third Conf. of On-farm Irrigation and Agroclimatology. Dokki, Egypt, 1 (37), 25- 27.

Abbas, J. A. (2007) Effect of Potassium Fertilization and Irrigation Intervals on Growth and Yield of Eggplant Solanum melnogenaL. Jordan J. Agric. Sci., 3 (3), 350-361.

Abd El-Aal, F. S., Abdel Mouty, M. M. and Ali,A. H. Egypt. J. Soil Sci., 57, No. 4 (2017)
(2008) Combined effect of irrigation intervals and foliar application of some antitranspirants on eggplant growth, fruits yield and its physical and chemical properties. Research J. Agricul. Biol. Sci., 4(5), 416-423.

Abdel Rahman, H.A., Ibrahim, A.A. and Elias, S.A. (1994) Effect of frequency and quantity of irrigation on growth and yield of cabbage (Brassica oleracea L.). European J. Agro., 3, 249-52

Abd El Rasool, S.F., Tawodros, H.W., Miseha, W.I. and Mahrous, F.N. (1971) Effect of irrigation and fertilization on water use efficiency by wheat Conf., Ain Shams Univ. Egypt.

AL-Rawahy, S.A., Abdel Rahman, H.A. and ALKalbani, M.S. (2004) Cabbage (Brassicaoleracea L.) response to soil moisture regime under surface and subsurface point and line applications. Int. J. Agri. Biol., 6 (6), 1093-1096.

Apel, K. and Hirt, H. (2004) Reactive oxygen species: Metabolism, oxidative stress, and signal transduction. Annual Review of Plant Biology, 55, 373-399.

Bahawireth, M.A.M. (2011) Physiological and yield performance of some okra and eggplant genotypes under water stress conditions. Ph.D. Thesis. Department Horti and vege. Crop. Assiut Univ., Egypt.

Black, C. A. (1965) Methods Of Soil Analysis. Part 2. Amer. Soci. of Agric. [NC] Publisher, Madison, Wisconsin.

Bruce W.B., Edmeades, G.O. and Baker,T.C. (2002) Molecular and physiological approaches to maize improvement for drought tolerance. Journal of Experimental Botany, 53: 13-25.

Byari, S. H. and Al-Sayed, A. R. (1999) The influence of differential irrigation regimes on five greenhouse tomato cultivars. 2.-The influence of differential irrigation regimes on fruit yield. Egyptian J. Horti. Sci., 26, 127-146.

Davenport, D. C, Uriu,K. and Hagan, R. M. (1974) Effects of film antitranspirants on growth. J. Exp. Bot. Vol. 25, No. 85,410-419.

El-Afifi, S.T.M., A. El-Sayed Hala, Farid, S.E.M. and Shalata, A.A.A. (2013) Effect of organic fertilization, irrigation intervals and some antitranspirants on growth and productivity of eggplant (Solanum melongena L.) Ph.D. Thesis. Department Vege. \& Flori. Department. Crop. Mansoura Univ., Egypt. 
Erdal, I. I., Ertek, A. Senyigit, U. and Koyuncu, M. A. (2007) Combined effects of irrigation and nitrogen on some quality parameters of processing tomato. World J. Agric. Sci., 3 (1), 57-62.

Fenton, R., Mansfield, T.A. and Jarvis , R.G. (1982) Stomatal movement. In JS McLaren, (Ed.) Chemical Manipulation of Crop Growth and Development. Butterworth Scientific, London, pp. 19-37

Fereres, E. and Soriano, M. A. (2007) Deficit irrigation for reducing agricultural water use. J. Exp. Bot., 58(2), 147-159.

Frank G. and Viets, J.R. (1967) Nutrient availability in relation to soil water. In Robert M. Hagan et al. Irrigation of Agricultural Lands. American Society of Agronomy, Publisher Madison, Wisconsin, USA.

Gaballah, M.S. and Moursy, M. (2004) Reflectants application for increasing wheat plant tolerance against salt stress. Pak. J. Biol. Sci. 7, 956-962.

Geerts, S. and Raes, D. (2009) Deficit irrigation as an onfarm strategy tm maximize crop water productivity in dry areas. Agricultural Water Management, 96, 1275-84.

Han, J.S. (1990) Use of antitranspirant epidermal coatings for plant protection in China. Plant Dis., 74, 263-266.

Hara, T. and Sonoda,Y. (1979) The role of macronutrients for cabbage-head formation, growth performance of a cabbage plant, and potassium nutrition in the plant. Soil Sci. Plant Nutr., 25 (1), 103-111.

Ibrahim, E.A., Abou El-Nasr,M.E. and Mohamed, M.R. (2011) Effect of irrigation levels and rice straw compost rates on yield, chemical composition and water use efficiency of cabbage (Brassicaoleraceae var. capitata L.). J. Plant Production, Mansoura Univ. (2), 413-424.

Iriti, M., Picchi,V., Rossoni, M., Gomarasca, S., Ludwig, N., Gargano, M. and Faoro,F. (2009) Chitosan antitranspirant activity is due to abscisic acid-dependent stomatal closure. Environmental and Experimental Botany, 66, 493-500

Israelson, O.W. and Hansen, V.E. (1962) Irrigation Principle and Practices. $3^{\text {rd }}$ ed.. John Willey \& Sons. New York.

Jackson, M.L. (1967) "Soil Chemical Analysis Advanced Course" Puble. By the auther, Dept. of Soils, Univ. of Wise., Madison 6, Wishensin, USA
Kage H., Kochler, M., Stutzel, H. (2004) Root growth and dry matter partitioning of cauliflower under drought stress conditions: measurement and simulation. European Journal of Agronomy, 20, 379-94.

Mahmoud, A.R. and Hafiz, M.M. (2002) Effect of irrigation intervals and sulphur application on the growth, yield and nutritive value of eggplant (Solanum melongena var. esculenta L.) Annals of Agric. Sci., Moshotohor, 40 (2), 1171-1182.

McKeown, A.W., Westerveld, S.M. and Bakker, C.J. (2010) Nitrogen and water requirements of fertigated cabbage in Ontario. Can. J. Plant Sci., 90 (1), 101-109.

Moftah, A.E. (1997) The response of soy bean plants, grown under different water regimes to antitranspiration application. Ann. Agric. Sci., 35, 263-292.

Nickell, L.G. (1982) Plant Growth Regulators: Agricultural Uses. Springer-Verlag, New York, pp 55-58

Parmar, H.C., Maliwal, G.L., Kaswala, R.R. and Patel, M.L. (1999) Effect of irrigation, nitrogen and spacing on yield of cabbage. Indian J. Horti., 56 (3), 256-258.

Pregle, E. (1945) "Quantitative Organic MicroAnalysis" 4th ed. J. Chudrial, London.

Sammis, T.W. and Wu, I.P. (1989) Deficit irrigation effects on head cabbage production. Agric. Water Manag., 16, 229-239.

Sammis, T.W., Kratky, B.A. and Wu, I.P. (1988) Effects of limited irrigation on lettuce and Chinese cabbage production. Irrig. Sci., 9, 187-198.

Sivakumar, D., Regnier,T. , Demoz, B. and Korsten, L. (2005) Effect of different post-harvesttreatments on overall quality retention in litchi fruit during low temperaturestorage. J. Hortic. Sci. Biotechnol. 80, 32-38.

Smittle D.A., Dickens, W.L. and Stansell, J.R. (1994) Irrigation regimes affect cabbage water use and yield. J. of the American Society for Horticultural Science. 119, 20-3.

Steel R.G.D. and Torrie J.H. (1998) Principles and Procedures of Statistics: A Biometric Approach R.G.Summerfield, A.H. Banting (Ed.), McGrawHill Press, New York, USA. 
Steinberg, S.L., McFarland, M.J. andWorthington, J.W. (1990) Antitranspirant reduces water use by peach trees following harvest. J. Am. Soc. Hortic. Sci., 115, 20-24.

Sutherland, F. andWalters, D.L. (2001) In vitro effects of film-forming polymers on the growth and morphology of Pyrenophora avenae and Pyricularia oryzae. J. Phytopathol.149, 621-624.

Sutherland, F. and Walters, D.L. (2002) Effect of filmforming polymers on infection ofbarley with the powdery mildew fungus Blumeria graminis f. sp. hordei. Eur. J.Plant Pathol. 108, 385-389.

Tambussi, E.A. and Bort, J. (2007) Water use efficiency in C3 cereals under Mediterranean conditions: a review of physiological aspects. Ann. Appl. Biol. 150, 307-321.

Tamer, A. N. (2014) Drought condition and management strategies in Egypt. Egyptian Meteorological Authority. $10 \mathrm{p}$.
Walters, D.L. (1992) The effects of three filmforming polymers, with and with out a polyamine biosynthesis inhibitor, on powdery mildew infection of barley seedlings. Ann. Appl. Biol. 120, 41-46.

Xu, C. and Leskover, D. (2014) Growth physiology and yield responses of cabbage to deficit irrigation. Horticulture Science, 41, 138-46.

Yadav , N. R. and Dashora, L. K. (2003) Shelflife of swat pepper (Capsicum annuum L.) cv. californiawonder as influenced by benzyladenine and vapor grad. Advances in Horticulture and Forestay, 9, 215-221.

(Received: 2 / 8/ 2017;

accepted: $6 / 9 / 2017)$ 